\title{
Monomorphic allele rs109231213 in 3'UTR PLAG1 gene in purebred of Bali cattle (Bos javanicus)
}

\author{
Sutikno Sutikno ${ }^{1, *}$, Jean Pierre Munyaneza ${ }^{2}$, Santiananda Arta Asmarasari ${ }^{3}$, and Jakaria \\ Jakaria $^{4}$ \\ ${ }^{1}$ Research Center for Biology, National Research and Innovation Agency (BRIN), Bogor 16911, \\ Indonesia \\ ${ }^{2}$ Division of Animal and Dairy Science, Department of Animal Science, College of Agriculture and \\ Life Sciences, Chungnam National University, Daejeon 34134, Korea \\ ${ }^{3}$ Indonesian Research Institute for Animal Production, Bogor 16720, Indonesia \\ ${ }^{4}$ Faculty of Animal Science, Bogor Agricultural University, Bogor 16680, Indonesia
}

\begin{abstract}
The mutation rs109231213 that is located in 3'UTR of PLAG1 gene is associated with the growth and body weight in several Bos taurus and Bos indicus breeds. This study aimed to identify SNP rs109231213 in Bali cattle (Bos javanicus). The study used 41 samples of Bali cattle. The PLAG1 gene polymorphism was analyzed using PCR and direct sequencing methods. PCR pimers were 5' - TTGCACAGAATCAGTGTGTC-3' and 5'AGCCTAACGTGGATCTATGG-3'. The results showed that primers successfully amplified the $331 \mathrm{bp}$ fragment at annealing $60^{\circ} \mathrm{C}$ that contained rs 109231213. SNP was monomorphic in Bali cattle with one allele $(G)$. This study concludes that rs109231213 in 3'UTR of PLAG1 gene can be used as specific marker in purebred of Bali cattle that have never been crossed with Bos taurus and Bos indicus.
\end{abstract}

\section{Introduction}

Bali cattle (Bos javanicus) are indigenous beef cattle in Indonesia that were domesticated from Banteng and are most preferred among small farmers [1-2]. Bali cattle are classified as beef cattle [3] with higher carcass percentages (42.1-51.1\%) [4], well adapted to tropical climates, good adaptations to intensive and extensive maintenance, and good reproductive ability [5]. The genetic diversity of Bali cattle is one of the important information in evaluating the genetic potential of livestock for the sake of conservation, sustainable use, and be developed into premium beef cattle.

Pleomorphic adenoma gene 1 (PLAG1) is associated with birth weight in Nellore and PO cattle [6-8], also with body size in Jersey, Friesian holstein, Simmental, Red Angus, Angus, Hereford, Limousin, Gelbvieh, Brangus, Maine Anjou cattle, Shorthorn, Charolais cattle [911] futhermore reproductive traits in Bos indicus and Bos taurus cattle [12-14]. Single nucleotide polymorphisms (SNPs) rs109231213 (chr 14:g.23.326.588, C > G forward strand

* Corresponding author: sutiknoabdulkadir@gmail.com 
based on ensembl) located on the 3'UTR of PLAG1 gene associated with height [15] and body weight in cattle [16]. Thus, the aim of this research was to identify rs109231213 polymorphism in Bali cattle.

\section{Materials and methods}

\subsection{DNA samples}

Total of DNA samples were used in this research are forty one. The sample of Bali cattle from the Superior Livestock Breeding and Livestock Forage Center of Denpasar (BPTUHPT Denpasar). DNA was obtained using the geneaid DNA extraction kit.

\subsection{PCR amplification and genotyping}

Primers were designed to amplify 331 base pairs fragment (Table 1). The SNP was accessed through ensembl (http://asia.ensembl.org/, SNP rs109231213, chromosome $14: 23.326 .588$ $\mathrm{C}>\mathrm{G}$ and gene sequence PLAG1 (access no: : ENSBTAG00000004022). This set of primers was made with software MEGA7 then evaluated by primer stats (www.bioinformatics.org) to test their suitability.

Table 1. A pair of primer to amplify 3'UTR of PLAG1 gene

\begin{tabular}{|l|c|c|c|}
\hline Primer & Sequence $\left(5^{\prime}-3^{\prime}\right)$ & Length & Ta $\left({ }^{\circ} \mathrm{C}\right)$ \\
\hline Forward & F: TTGCACAGAATCAGTGTGTC & \multirow{2}{*}{$331 \mathrm{bp}$} & 60 \\
\hline Reverse & R: AGCCTAACGTGGATCTATGG & & \\
\hline
\end{tabular}

GenBank access No. NC_037341 fragment position PCR : 23.326.380-23.375.751 (331 bp) SNP position rs $109231213(\mathrm{chr} 14: 23.326 .588, \mathrm{C}>\mathrm{G})$ ensembl rs 109231213

The PCR mix volume was $25 \mu \mathrm{L}$, containing of $1 \mu \mathrm{L}$ DNA template $(50 \mathrm{ng}), 0.3 \mu \mathrm{L}$ primer forward and reverse (5 pmol), $12.5 \mu \mathrm{l}$ GoTaq ${ }^{\circledR}$ Green Master Mix (1x Promega), and $10.9 \mu \mathrm{L}$ nuclease-free water. PCR mix run using Applied Biosystem thermal cycler, with condition pre-denaturation was at $95^{\circ} \mathrm{C}$ for $5 \mathrm{~min}$, and amplifiction for 35 cycles (denaturation at $95^{\circ} \mathrm{C}$ for $10 \mathrm{~s}$, annealing at $60^{\circ} \mathrm{C}$ for $20 \mathrm{~s}$, extension at $72^{\circ} \mathrm{C}$ for $30 \mathrm{~s}$, and final elongation at $72^{\circ} \mathrm{C}$ for $5 \mathrm{~min}$. The PCR products visualized by electrophoresis using $2 \%$ agarose gel and sequenced by First BASE Laboratories Selangor, Malaysia.

\subsection{Data analysis}

The frequencies genotype and allele were calculated by software Popgene 3.2 [17].

\section{Results and discussion}

PLAG1 gene located on 5'UTR of Bali cattle was $100 \%$ successfully amplified at $60^{\circ} \mathrm{C}$ of annealing temperature (Fig. 1). The length of PCR product was $331 \mathrm{bp}$. Sequencing of PLAG1 gene located on 5'UTR was monomorphic in Bali cattle, only one allele C was found (Fig. 2). 


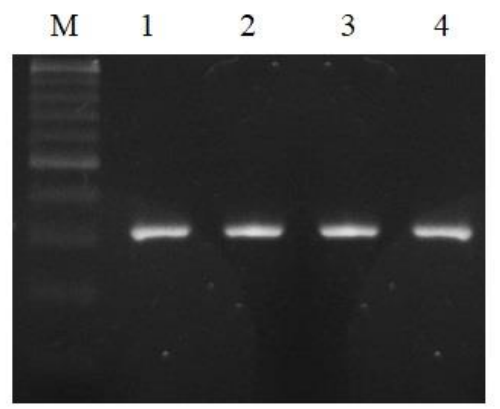

$331 \mathrm{bp}$

Fig. 1. Visualization of PCR product, $M$ was marker $100 \mathrm{bp}$, line 1,2,3, 4 were analyzed samples.
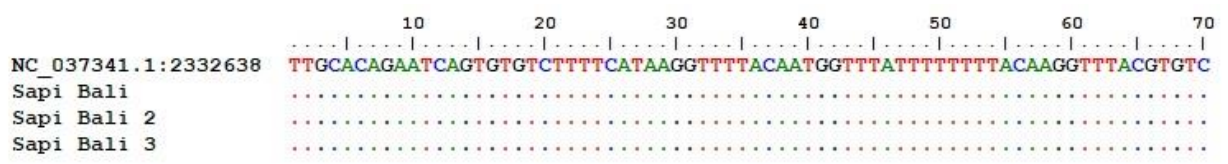

NC $037341.1: 2332638$

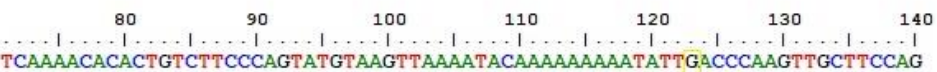
Sap̄i Bali

Sapi Bali 2

Sapi Bali 3

NC_037341.1:2332638

Sap̃i Bali

Sapi Bali 2

Sapi Bali 3

NC_037341.1:2332638

Sap̄i Bali

Sapi Bali 2

Sapi Bali 3

NC 037341.1:2332638

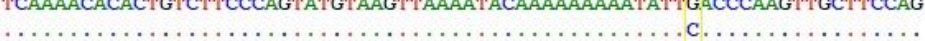

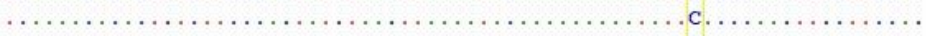
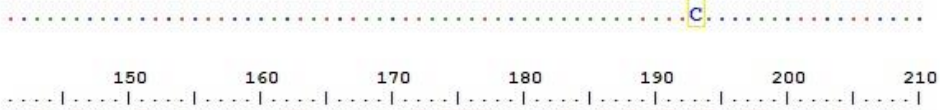
CCTACTGAGATCCAGGCGACATTGGAGGAGATCTTTTAGACGTCATGTATTCTTCATTAGCAATGGCAGA

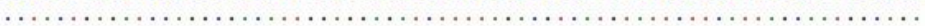
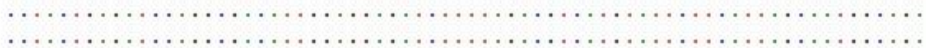

Sapi Bali

Sapi Bali 2

Sapi Bali 3

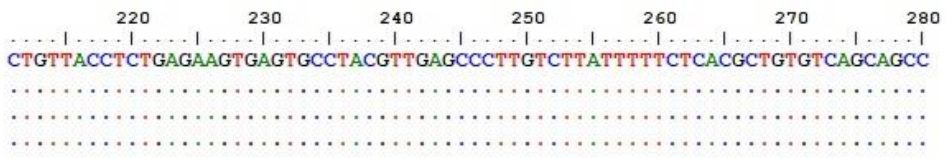

Fig. 2. Visualisazion of the 3'UTR sequence alignment of the PLAG1 gene in Bali cattle

The rs109231213 was polymorphic in Bos taurus, Bos indicus and Tropical Composite cattle with frequency $\mathrm{C}$ allele was $0.96,0.52,0.68$ and $\mathrm{G}$ allele $0.04,0.48,0,32$, respectively. The $\mathrm{C}$ allele increased hip height, weight, net food intake, age at puberty, fat depth and decreased IGF-I concentration in blood [9-11,18]. The results show that Bali cattle have genetic potential to be developed into premium beef cattle based on this SNP. Futhermore, PLAG1 became as a key regulator of mammalian growth [6-8].

Previously study of rs 109231213 reported that $\mathrm{G}$ is ancestral allele and $\mathrm{C}$ is derived allele. The $\mathrm{C}$ allele was introgressed from Bos taurus to Brahman cattle during the grading up of Brahman cattle [19]. Introgression is the transfer of genetic information from Bos taurus to Brahman cattle as a result of hybridization between them and repeated backcrossing. In this study Bali cattle did not have the $\mathrm{G}$ allele. The results show that Bali cattle have never been 
crossed with Bos taurus (i.e. Hereford, Angus, Shorthorn) and Bos indicus (i.e. Brahman, PO).

\section{Conclusion}

SNP rs109231213 is monomorphic in Bali cattle (C allele). Bali cattle have genetic potential to be developed into premium beef cattle based on this SNP. Furthermore, providing information that Bali cattle have never been crossed with Bos indicus, Bos taurus, and composite cattle.

\section{References}

1. P. Gardner, S. Hedges, S. Pudyatmoko, T. N. E. Gray, R. J. Timmins, The IUCN Red List of Threatened Species 2016: e.T2888A46362970 (2016)

2. H. Martojo, Reprod. Dom. Anim. 47, 10-14 (2012)

3. J. Jakaria, H. Khasanah, R. Priyanto, M. Baihaqi, M. F. Ulum, J. Indones. Trop. Anim. Agric. 42, 59-65 (2017)

4. H. Hafid, Nuraini, Inderawati, W. Kurniawan, IOP Conf. Ser. Earth Environ. Sci. 119, 012043 (2018)

5. Sutarno, A. D. Setyawan, Biodiversitas 16, 327-354 (2015)

6. H. Hartati, Y. T. Utsunomiya, T. S. Sonstegard, J. F. Garcia, J. Jakaria, M. Muladno, BMC Genet. 16, 75 (2015)

7. Y. T. Utsunomiya, A. S. do Carmo, R. Carvalheiro, H. H. R. Neves, M. C. Matos, L. B. Zavarez, A. M. P. O’Brien, J. Sölkner, J. C. Mc Ewan, J. B. Cole, C. P. Van Tassell, F. S. Schenkel, M. V. G. B. da Silva, L. R. P. Neto, T. S. Sonstegard, J. F. Garcia, BMC Genet. 14, 52 (2013)

8. M. D. Littlejohn, T. Grala, K. Sanders, C. Walker, G. Waghorn, K. MacDonald, W. Coppieters, M. Georges, R. Spelman, E. Hillerton, S. Davis, R. Snell, Anim. Genet. 43, 591-594 (2012)

9. L. Karim, H. Takeda, L. Lin, T. Druet, J. A. C. Arias, D. Baurain, N. Cambisano, S. R. Davis, F. Farnir, B. Grisart, B. L. Harris, M. D. Keehan, M. D. Littlejohn, R. J. Spelman, M. Georges, W. Coppieters, Nat. Genet. 43, 405-413 (2011)

10. M. Saatchi, R. D. Schnabel, J. F. Taylor, D. J. Garrick, BMC Genom. 15, 442 (2014)

11. M. R. S. Fortes, K. Kemper, S. Sasazaki, A. Reverter, J. E. Pryce, W. Barendse, R. Bunch, R. McCulloch, B. Harrison, S. Bolormaa, Y. D. Zhang, R. J. Hawken, M. E. Goddard, S. A. Lehnert, Anim. Genet. 44, 636-647 (2013)

12. Y. T. Utsunomiya, A. S. Carmo, H. H. R. Neves, R. Carvalheiro, M. C. Matos, L. B. Zavarez, P. K. R. K. Ito, A. M. P. O’Brien, J. S. lkner, L. R. Porto-Neto, F. S. Schenkel, J. McEwan, J. B. Cole, M. V. G. B. da Silva, C. P. V. Tassell, T. S. Sonstegard, J. F. Garcia, Plos One 9, e88561 (2014)

13. M. R. S. Fortes, A. Reverter, M. Kelly, R. McCulloch, S. A. Lehnert, Andrology 1, 644650 (2013)

14. M. R. S. Fortes, S. A. Lehnert, S. Bolormaa, C. Reich, G. Fordyce, N. J. Corbet, V. Whan, R. J. Hawken, A. Reverter, Anim. Prod. Sci. 52, 143-150 (2012)

15. J. L. Zhong, J. W. Xua, J. Wanga, Y. Wena, H. Niub, L. Zheng, H. Hea, K. Penga, P. Hea, S. Shia, Y. Huang, C. Leia, R. Danga, X. Lana, X. Qid, H. Chena, Y. Huang, Gene 689, 166-171 (2019) 
16. Y. T. Utsunomiya, M. Milanesi, A. T. H. Utsunomiya, R. B. P. Torrecilha, E. S. Kim, M. S. Costa, T. S. Aguiar, S. Schroeder, A. S. do Carmo, R. Carvalheiro, H. H. R. Neves, R. C. M. Padula, T. S. Sussai, L. B. Zavarez, R. S. Cipriano, M. M. T. Caminhas, G. Hambrecht, L. Colli, E. Eufemi, P. Ajmone-Marsan, D. Cesana, M. Sannazaro, M. Buora, M. Morgante, G. Liu, D. Bickhart, C. P. Van Tassell, J. Sölkner, T. S. Sonstegard, J. F. Garcia, Sci. Rep. 7, 17140 (2017)

17. F. C. Yeh, R. C. Yang, T. Boyle, Popgene Version 1.31: Microsoft Window-Based Freeware for Population Genetics Analysis (University of Alberta, Edmonton, Canada, 1999)

18. M. R. S. Fortes, K. Kemper, S. Sasazaki, A. Reverter, J. E. Pryce, W. Barendse, R. Bunch, R. McCulloch, B. Harrison, S. Bolormaa, Y. D. Zhang, R. J. Hawken, M. E. Goddard, S. A. Lehnert, Anim. Genet. 44, 636-647 (2013)

19. L. Karim, H. Takeda, L. Lin, T. Druet, J. A. C. Arias, D. Baurain, N. Cambisano, S. R. Davis, F. Farnir, B. Grisart, B. L. Harris, M. D. Keehan, M. D. Littejohn, R. J. Spelman, M. Georges, W. Coppieters, Nat. Genet. 43, 405-413 (2011) 